\title{
NUDCD1 knockdown inhibits the proliferation, migration, and invasion of pancreatic cancer via the EMT process
}

\author{
Chunling Shi ${ }^{1}$, Min Weng ${ }^{2}$, Hengyue Zhu ${ }^{2}$, Yangyang Guo ${ }^{2}$, Dongdong $\mathrm{Xu}^{1}$, Hairu Jin ${ }^{1}$, \\ Binshuang $\mathrm{Wei}^{1}$, Zhensheng Cao ${ }^{1}$ \\ ${ }^{1}$ School of Stomatology, Wenzhou Medical University, Wenzhou 110013, China \\ ${ }^{2}$ Key Laboratory of Diagnosis and Treatment of Severe Hepato-Pancreatic Diseases of Zhejiang Province, The First \\ Affiliated Hospital of Wenzhou Medical University, Wenzhou 325000, China
}

Correspondence to: Hairu Jin, Binshuang Wei, Zhensheng Cao; email: 365023543@qq.com, https://orcid.org/0000-00024170-9492; 402478121@qq.com, https://orcid.org/0000-0002-5339-2226; 527990849@qq.com, https://orcid.org/00000003-2367-6639

Keywords: pancreatic cancer, NUDCD1, EMT, apoptosis, proliferation Abbreviations: PC: Pancreatic cancer; qRT-PCR: Quantitative real-time polymerase chain reaction; RTCA: Real-time cell analysis; EMT: Epithelial-mesenchymal transition

Received: September 17, 2020 Accepted: April 29, 2021

Published: July 29, 2021

Copyright: (C) 2021 Shi et al. This is an open access article distributed under the terms of the Creative Commons Attribution License (CC BY 3.0), which permits unrestricted use, distribution, and reproduction in any medium, provided the original author and source are credited.

\section{ABSTRACT}

NudC domain containing 1 (NUDCD1) is an oncoprotein frequently activated or upregulated in various human cancers, but its role in pancreatic cancer (PC) remains unknown. Thus, we aimed to determine the function and mechanism of NUDCD1 in PC. We employed Western blot and quantitative real-time polymerase chain reaction to assess NUDCD1 expression in cells and PC tissues. NUDCD1 was knocked down in Patu8988 and PANC-1 cells. We conducted real-time cell analysis, wound healing assay, transwell assay and colony formation assay to evaluate the metastatic and proliferative abilities of PC cells. Western blot was conducted to assess the expression of markers associated with apoptosis and epithelial-mesenchymal transition (EMT). Also, we established a tumor xenograft model to determine the role of NUDCD1 in vivo. NUDCD1 was overexpressed in PC tissues and cells. NUDCD1 knockdown suppressed the invasion, migration, and proliferative abilities of the cells and induced PC cell apoptosis. The specific mechanism of NUDCD1 was related to the modulation of the EMT process. Data obtained from in vivo experiments revealed that NUDCD1 knockdown inhibited the tumor growth, proliferation, and metastasis by modulating the EMT and inducing the apoptosis of PC cells.

\section{INTRODUCTION}

Pancreatic cancer (PC) is a cancer that is highly resistant to chemotherapy, grows rapidly, and undergoes early distant metastasis $[1,2]$. Patients with PC have extremely poor prognosis because of distant metastasis and the high frequency of tumor recurrence [3, 4]. Although multiple genes in tumor recurrence and metastasis have been identified, the underlying mechanism of PC metastasis remains unclear. Looking for reliable biomarkers for recurrence and metastasis will greatly benefit patients with PC and may provide novel gene targets for PC treatment.

NudC domain containing 1 (NUDCD1, also known as CML66 or OVA66) is an oncoprotein that is frequently activated or upregulated in various human cancers and well known as an important cancer antigen [5-7]. NUDCD1 is involved in the regulation of different biological processes, such as apoptosis and immune responses [8]. NUDCD1 overexpression enhances the proliferation, invasion, and survival of 
HO-8910 cells [9]. Although current evidence suggests that NUDCD1 may act as an oncoprotein, its expression and molecular mechanisms in $\mathrm{PC}$ metastasis remain unclear.

We hypothesized that NUDCD1 plays a crucial function during the progression of PC. We measured NUDCD1 expression in human PC tissues and determined its contribution to $\mathrm{PC}$ proliferation, invasion, and migration in vitro and in vivo to test our hypothesis. Our data revealed that NUDCD1 is upregulated in PC cells and tissues and plays a major metastatic role in growth, epithelial-mesenchymal transition (EMT), and PC cell apoptosis.

\section{MATERIALS AND METHODS}

\section{Cell culture and experimental reagents}

Human PC cell lines PANC-1, CFPAC-1, and Patu8988 (ATCC, Manassas, VA, USA) were grown in Dulbecco's modified Eagle medium (DMEM; Invitrogen, Carlsbad, CA) that contained $10 \%$ fetal bovine serum and 1\% penicillin-streptomycin (Sigma, Taufkirchen, Germany). Primary antibodies for NUDCD1 (Ab126902, 1:1000) and GAPDH (Ab8245, 1:2000) were obtained from Abcam. Antibodies against human Bcl-2 (12789-1-AP, 1:1000), Bax (50599-2-Ig, 1:1000) and Caspase 3 (19677-1-AP, 1:1000) were purchased from Proteintech. Antibodies against vimentin (AF7013, 1:1000), N-cadherin (AF4039, 1:1000), Cleaved-Caspase 3 (AF7022, 1:1000) and Ecadherin (AF0131, 1:1000) were purchased from Affinity Biosciences.

\section{SiRNA transfection}

The cell lines PANC-1 and Patu8988, which highly express NUDCD1, were screened from various pancreatic cell lines by Western blot and quantitative real-time polymerase chain reaction (qRT-PCR). SiRNAs that target NUDCD1 were synthesized by GenePharma (Shanghai, China). The primer sequences of the siRNAs were as follows: NUDCD1 siRNA1, 5'-GGUGCUAGGUAGAAAGUUAGG-3' (sense) and 5'-UAACUUUCUACCUAGCACCUC-3' (antisense); NUDCD1 siRNA2，5'-GAUAGUACUAGCAAGUA UACU-3' (sense) and 5'-UAUACUUGCUAGUAC UAUCUU-3' (antisense); NUDCD1 siRNA3, 5'-AG AUAUGUAUCUUAUAUAAAC-3' (sense) and 5'-UU AUAUAAGAUACAUAUCUUG-3' (antisense). We transfected the PANC-1 and Patu8988 cells with siRNAs using Lipofectamine 3000 (Life Technologies) as per the instructions of the manufacturer for $48 \mathrm{~h}$. The efficiency of siRNA transfection was assessed by qRT-PCR.

\section{Immunohistochemistry (IHC) assay}

We conducted IHC according to the methods described previously [10]. In brief, sections were fixed in formalin, embedded in paraffin, and stained with antibody against NUDCD1 (1:100). The primary antibody was substituted with preimmune $\operatorname{IgG}$ serum in negative controls.

\section{Real-time cell analysis (RTCA)}

The cell proliferation of the NUDCD1 knocked down Patu8988 and PANC-1 cells was measured by a commercially available RTCA kit as per the manufacturer's protocol. We first determined the background level by loading $200 \mu \mathrm{L} /$ well of culture medium (DMEM with 10\% FBS) into a 16-well Eplate. Cells (about 10,000 cells/well) were seeded in the E-plate and cultured for $24 \mathrm{~h}$. Cell index was measured every $15 \mathrm{~min}$. Changes in the number and activity of cells were reflected by the detection of the cellular index of reactive cell activity.

\section{Colony formation assay}

We seeded PC cell lines (with or without siRNA transfection) into 6-well plates $(600$ cells/well $)$. Subsequently, we cultured the cells at $37^{\circ} \mathrm{C}$ in complete medium in a humidified atmosphere with $5 \% \mathrm{CO}_{2}$. The medium was refreshed at a 2-day interval. The culture was maintained for 2 weeks, and the colonies were stained with crystal violet solution before the number of clones was counted. This data was then used to determine the cell proliferation rate.

\section{Flow cytometry analysis}

An apoptosis detection kit (Pharmingen, San Diego, CA, USA) was used to conduct propidium iodide (PI) and annexin V-fluorescein isothiocyanate (FITC) staining to determine the rate of cell apoptosis. Subsequently, flow cytometric analysis was performed following NUDCD1 knockdown in PANC-1 and Patu8988 cells as per the manufacturer's protocol. In brief, the cells $\left(5 \times 10^{5}\right.$ cells/well) were grown for $24 \mathrm{~h}$ and then stained with Annexin V. Next, the cells were rinsed with PBS, then resuspended in a binding buffer $(100 \mathrm{~mL})$ with $5 \mu \mathrm{L}$ FITC and $5 \mu \mathrm{L}$ PI, and incubated for $30 \mathrm{~min}$ in the dark. Finally, a FACScalibur flow cytometer was used to analyze the cells after adding 400 $\mu \mathrm{L}$ of binding buffer.

\section{Wound healing assay}

Wound healing assay was conducted to evaluate the migration ability of the tumor cells. After transfection, 
the cells were seeded into 6-well plates and incubated for $48 \mathrm{~h}$. A sterile pipette tip $(100 \mu \mathrm{L})$ was used to scratch the surface of a monolayer to create wounds. Debris and keratinocytes were washed off with PBS, and the medium was substituted with serum-free DMEM. Finally, we examined the wound area and took photographs at 0 and $24 \mathrm{~h}$ to determine the number of migrated cells.

\section{Transwell invasion assay}

Transwell invasion assay was used to assess the invasive capacity of the cells. In brief, the PANC-1 and Patu8988 cells with serum-free DMEM medium were seeded into the upper chamber after NUDCD1 knockdown. Matrigel (1:8 diluent of $50 \mathrm{mg} / \mathrm{L}$ ) was coated on the upper surface of the bottom membrane of the Transwell chamber and dried at $4^{\circ} \mathrm{C}$. We filled the bottom wells with complete DMEM. The cells in the upper chamber were removed after $24 \mathrm{~h}$ of incubation. The cells that moved via the Matrigel matrix membrane were fixed in $4 \%$ paraformaldehyde and stained with $0.1 \%$ crystal violet. Finally, the cells were visualized under a microscope and photographed. We randomly selected five fields and counted the number of cells in each field.

\section{Gene expression analysis}

TRIzol (Invitrogen) was used to extract total RNA from the knocked down cells and control cells. The RNA was further purified using the RNeasy Mini Kit and RNasefree DNase Set (Qiagen, Valencia, CA, USA) as per the instructions of the manufacturer. Next, the RNA was reverse-transcribed to cDNA using a cDNA synthesis kit (Promega M-MLV cDNA) as per the protocol of the manufacturer. Subsequently, we conducted qRT-PCR assay to assess NUDCD1 mRNA expression. $\beta$-actin served as the internal control for reference. The following are the sequences of the primers used: NUDCD1， 5'-CAGCCCTTTGTGAGTGCCTTCG-3' (forward) and 5'-GTCCTACTTGCCTGCCTTCCT TTC-3' (reverse); $\beta$-actin, 5'-TGACGTGGACATCCGC AAAG-3' (forward) and 5'-CTGGAAGGTGGACA GCGAGG-3' (reverse).

\section{Western blot analysis}

We performed Western blot according to the methods described by Nina Kočevar et al [11]. The cells were removed from the culture plates, resuspended in PBS, and collected via centrifugation. Cell lysis was done using lysis buffer at $4^{\circ} \mathrm{C}$ for $20 \mathrm{~min}$. We sonicated and extracted the cells via 30-min high-speed centrifugation at $4{ }^{\circ} \mathrm{C}$ to remove cell debris. Protein assay system (BioRad, Hercules, CA, USA) was employed to evaluate the protein concentration. Subsequently, the proteins (50 $\mathrm{mg}$ ) were resolved by sodium dodecyl sulfatepolyacrylamide gel electrophoresis and transferred onto nitrocellulose membranes. The membranes were incubated with antibodies specific for the desired proteins. The proteins were detected using an enhanced chemiluminescence Western blot kit (Pierce, Rockford, IL, USA) as per the manufacturer's protocol.

\section{Tumor formation in nude mice}

Twelve BALB/CA nude mice (weight, 16-20 g; age, 6-8 weeks) were randomized into three groups: Si-NC, Si-RNA1, and Si-RNA2. We injected the cell suspension $\left(100 \mu \mathrm{L}, 5 \times 10^{6}\right.$ cells $)$ subcutaneously into the right axilla of each mice. Tumorigenesis in the mice was assessed weekly. The mice were sacrificed via $\mathrm{CO} 2$ asphyxiation 4 weeks postinjection, and the tumors were extracted to determine their weight and volume (volume $=1 / 2 \times$ length $\times$ width $^{2}$ ).

\section{Data analysis}

Data were analyzed using GraphPad software. Student's t-test was used to assess the difference between the experimental and control groups. Statistical significance was set at $\mathrm{p}<0.05$.

\section{RESULTS}

\section{Expression and clinical importance of NUDCD1 in pancreatic ductal adenocarcinoma (PDAC)}

Herein, we employed the Gene Expression Profiling Interactive Analysis server (http://gepia.cancer-pku.cn/) to mine and analyze NUDCD1 expression data according to the Genotype-Tissue Expression and The Cancer Genome Atlas databases. Based on the analysis, the levels of NUDCD1 mRNA were overtly higher in PDAC tissues in comparison to normal pancreatic tissues (Figure 1A). According to log-rank test, the upregulation of NUDCD1 was significantly related to overall survival $(\mathrm{p}=0.041)$ and disease-free survival ( $\mathrm{p}=0.037$; Figure 1B, 1C). Furthermore, the IHC results revealed that NUDCD1 was overexpressed in PDAC tissues relative to matched adjacent tissues (Figure 1D). We determined the NUDCD1 expression in many PC cell lines versus normal pancreatic cell line HPNE via qRT-qPCR and Western blot. Based on the results, NUDCD1 was markedly upregulated in PANC-1, CFPAC-1, and Patu8988 cells (Figure 1E, 1F), especially in Patu8988 and PANC-1 cells. Collectively, these findings indicated that NUDCD1 is aberrantly overexpressed in PDAC. 


\section{NUDCD1 knockdown inhibits the proliferation and colony formation of $\mathrm{PC}$ cells}

Considering that NUDCD1 is usually upregulated in PC, we speculated that NUDCD1 could facilitate PC progression. PANC-1 and Patu8988 cell lines, which highly express NUDCD1, were used to examine the role of NUDCD1 in the pathogenesis of PC. Thus, siRNA1, siRNA2, and siRNA3 were used to knock down NUDCD1 expression. Obviously, siRNA1 and siRNA2 reduced NUDCD1 expression by half or more (Figure 2A, 2B). The RTCA results showed that the silencing of NUDCD1 remarkably inhibited the proliferation of
PANC-1 and Patu8988 cells (Figure 2C, 2D). In addition, NUDCD1 gene knockdown obviously reduced the colony formation of PC cells (Figure 2E-2H).

\section{NUDCD1 gene silencing triggers $P C$ cell apoptosis in vitro}

Considering the data from cell proliferation and colony formation assay, we speculated that NUDCD1 could have a role in the apoptosis of PC cells. Thus, we performed flow cytometry to examine the apoptosis of cells transfected with si-NUDCD1. As shown in Figure 3A-3D, NUDCD1 knockdown remarkably increased
A

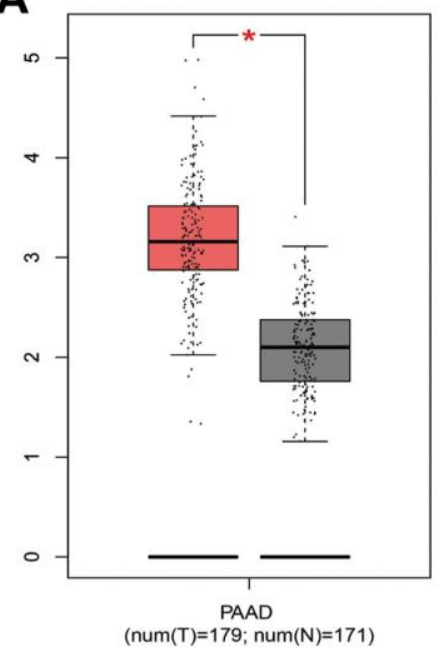

D

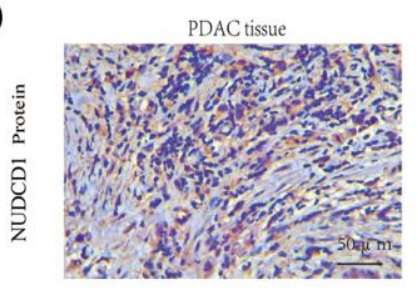

B
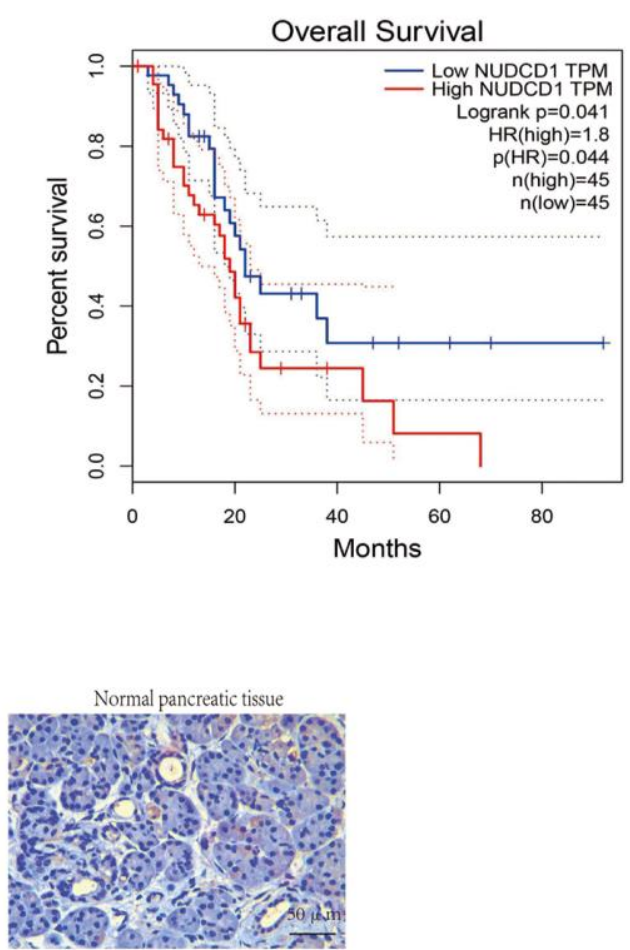

C

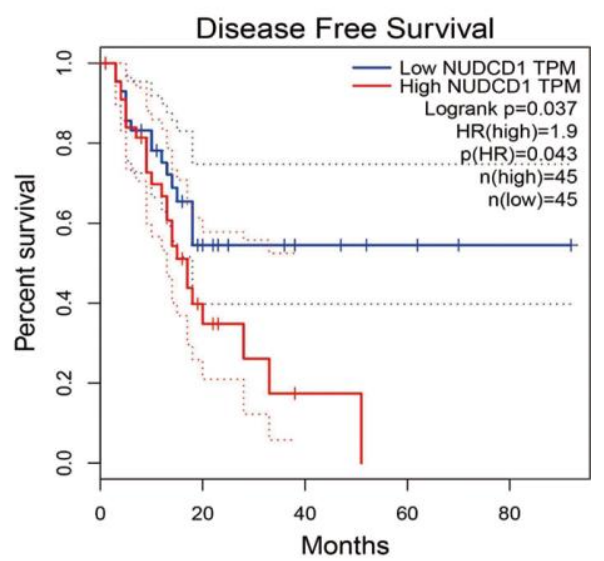

$\mathbf{E}$

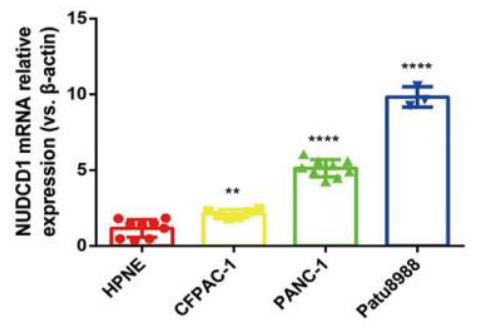

$\mathbf{F}$

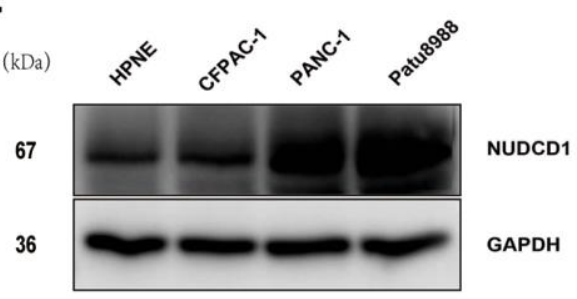

G

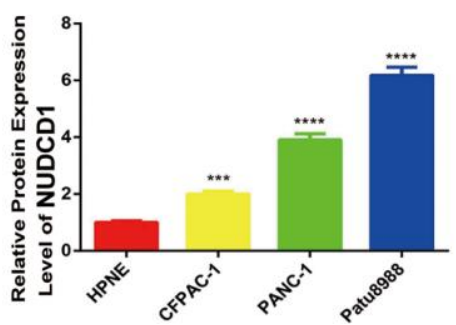

Figure 1. Expression and roles of NUDCD1 in PC. (A) NUDCD1 mRNA expression levels were significantly higher in PDAC tissues ( $\mathrm{n}=$ 179) than in normal pancreatic tissues $(n=171)$. (B, C) NUDCD1 upregulation was significantly correlated with shorter overall survival and shorter disease-free survival ( $p=0.041$ and 0.037 , respectively). (D) NUDCD1 was overexpressed in PDAC tissues relative to adjacent tissues as detected by IHC. (E) NUDCD1 upregulation was observed in different PC cell lines by qRT-PCR. (F, G) High NUDCD1 expression was observed in different PC cell lines by Western blot. ${ }^{*} p<0.05,{ }^{*} p<0.01,{ }^{*} * *<<0.001,{ }^{* * * *} p<0.0001$. 
A

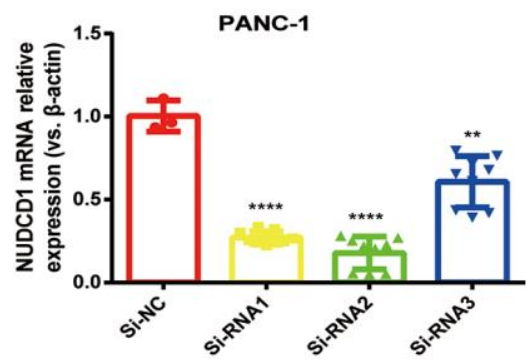

C

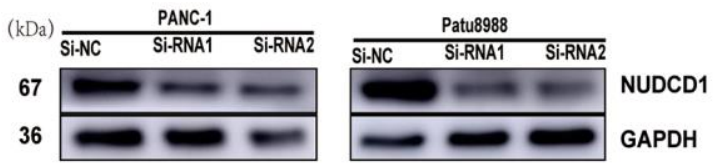

F

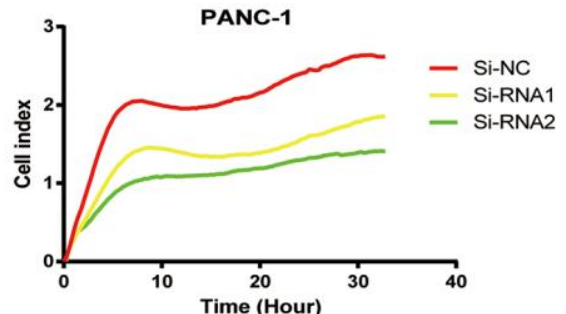

H

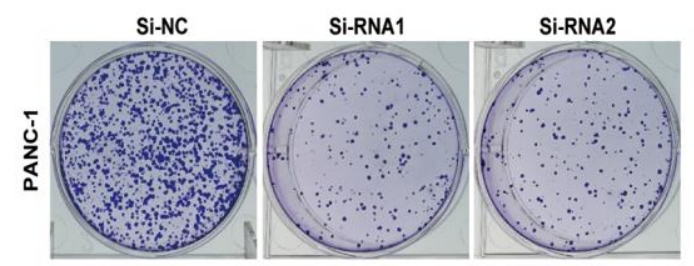

J

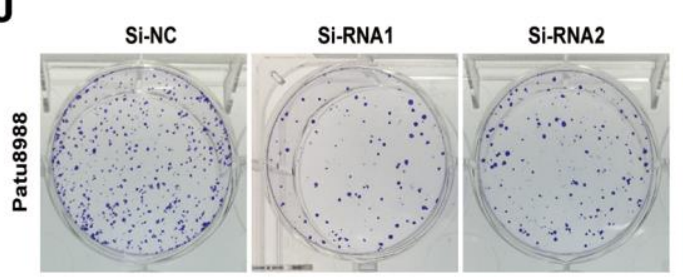

L
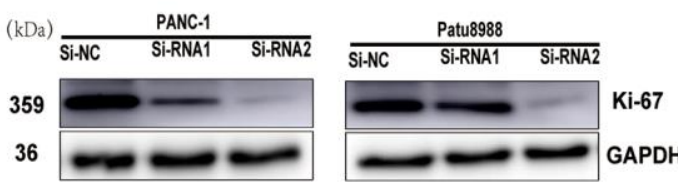

B
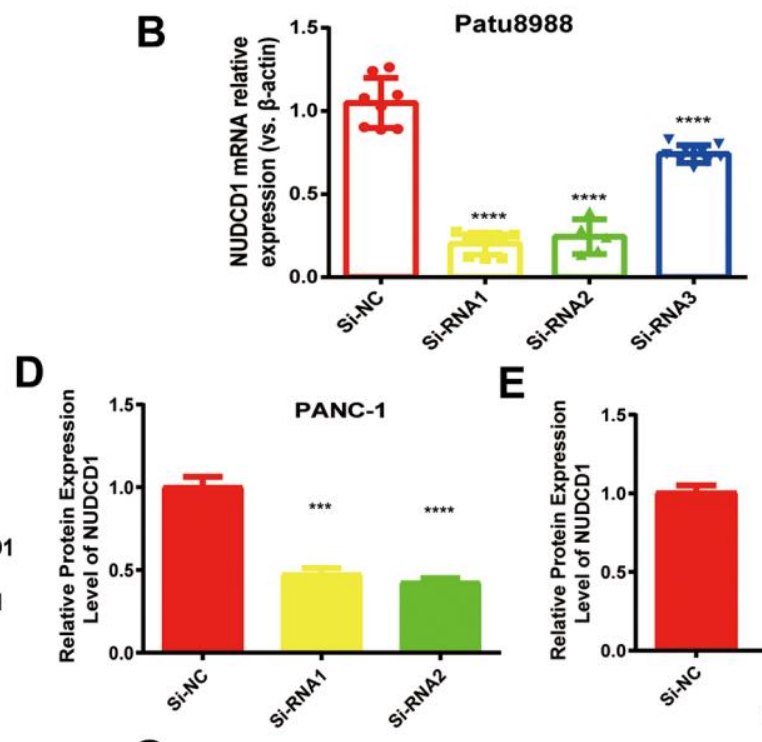

E

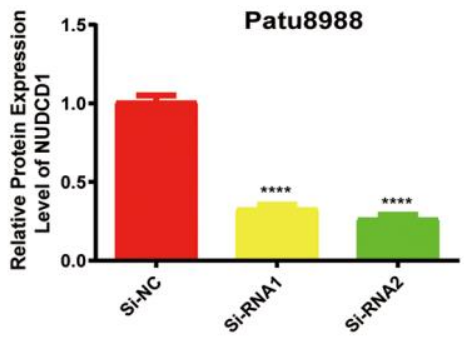

Patu8988

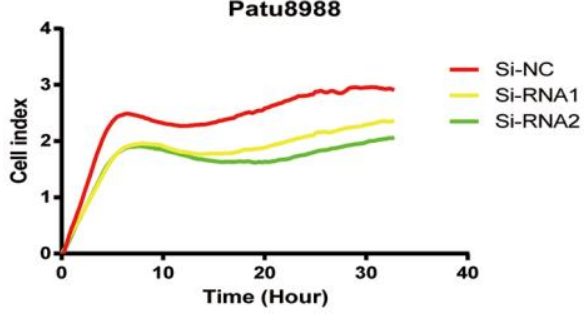

I

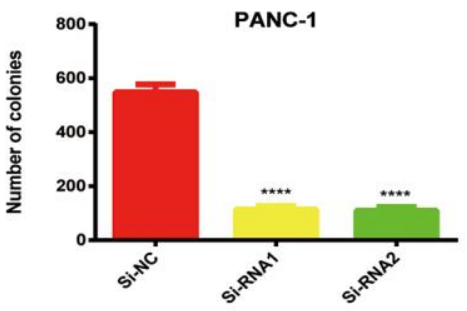

K

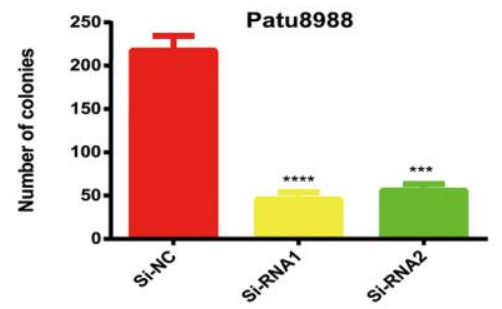

M

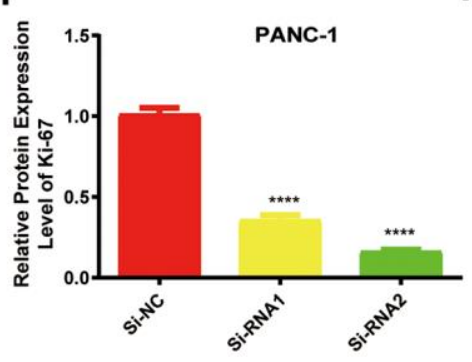

Figure 2. NUDCD1 knockdown inhibited the proliferation of PC cells. (A, B) The interference effects of three siRNAs on NUDCD1 were confirmed by qRT-PCR. (C-G) NUDCD1 knockdown inhibited the proliferation of PANC-1 and Patu8988 cells. (H-K) NUDCD1 knockdown inhibited the colony formation of Patu8988 and PANC-1 cells. (L-N) Western blot analysis showed that NUDCD1 knockdown decreased the expression of Ki-67 in PANC-1 and Patu8988 cells. ${ }^{* *} p<0.01, * * * p<0.001, * * * * p<0.0001$. 


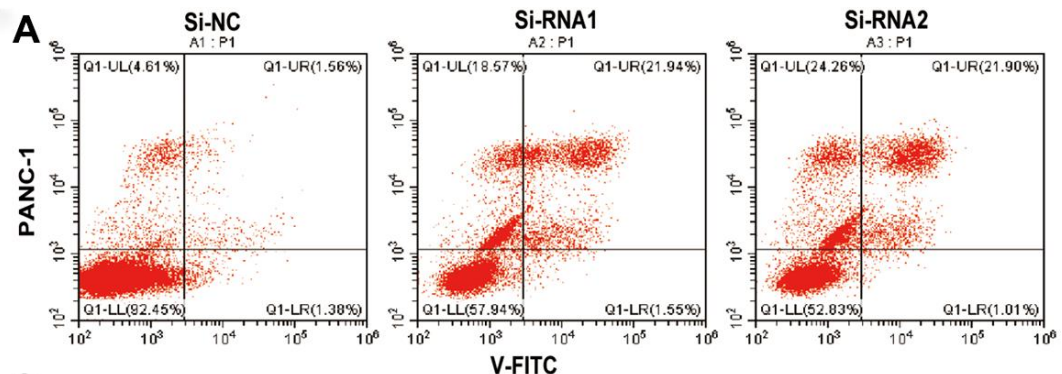

B
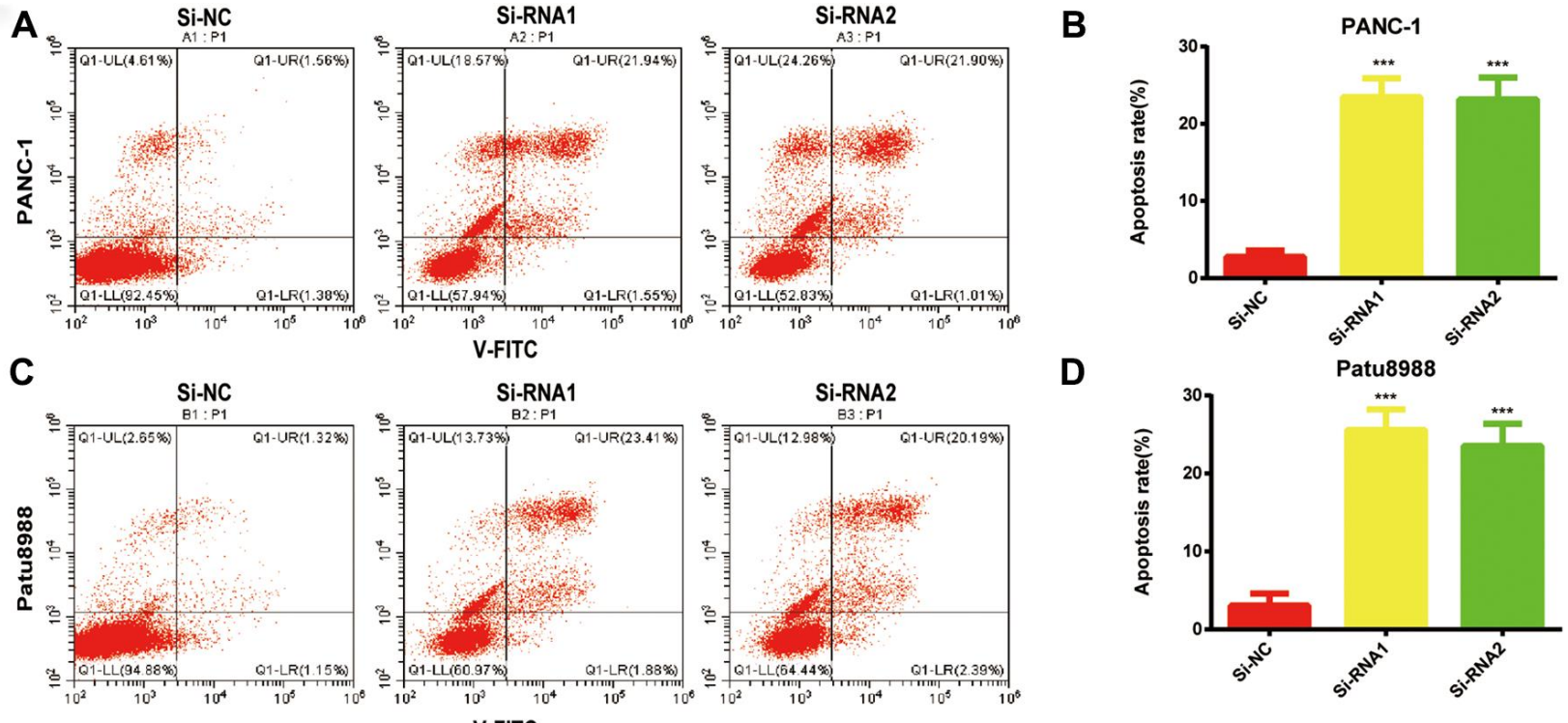

D
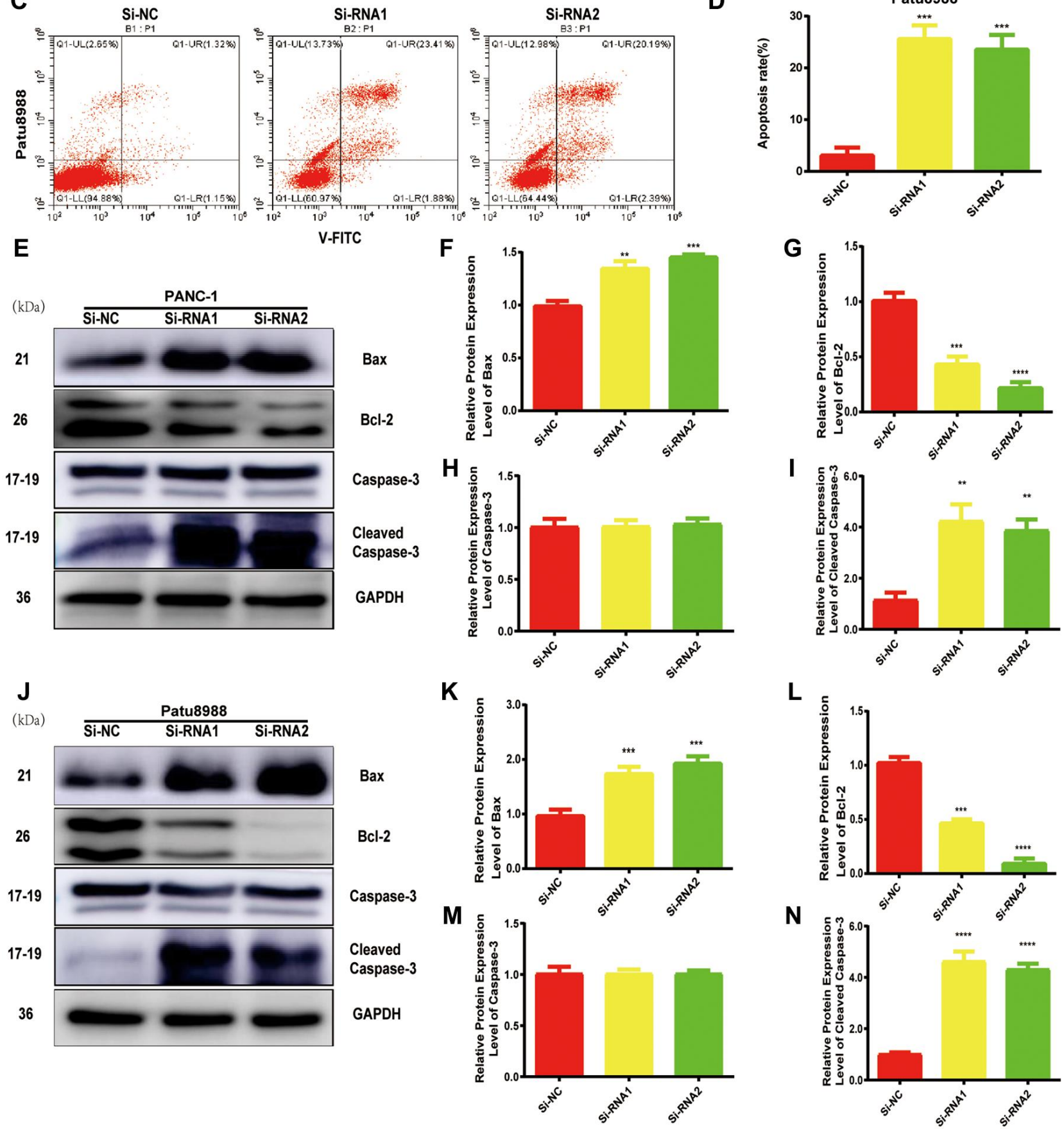

N

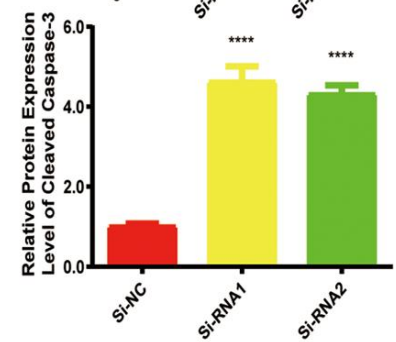

Figure 3. NUDCD1 knockdown induced the apoptosis of PC cells. (A-D) Flow cytometric analysis revealed that NUDCD1 knockdown increased the rate of apoptosis in Patu8988 and PANC-1 cells. (E-N) Western blot analysis showed that NUDCD1 knockdown decreased the expression of Bcl-2 and increased the expression of Bax and Cleaved Caspase-3 in PANC-1 and Patu8988 cells. ${ }^{* *}$ p $<0.01,{ }^{* * *}<<0.001$, $* * * * p<0.0001$. 
the rate of apoptosis in PC cells (Patu8988 and PANC-1) relative to the equivalent $\mathrm{Si}-\mathrm{NC}$ group. Furthermore, we detected the levels of apoptosisrelated proteins and found that NUDCD1 knockdown upregulated $\mathrm{Bax}$ and downregulated Bcl-2 (Figure $3 \mathrm{E}-3 \mathrm{~J})$

\section{NUDCD1 knockdown reduces the invasion and migration abilities of $P C$ cells}

Considering the data from our colony formation and cell proliferation assays, NUDCD1 may influence the invasion and migration abilities of PC cells. Expectedly, we found that that NUDCD1 knockdown considerably suppressed the migration capacity of Patu8988 and PANC-1 cells relative to the control group (Figure 4A-4D). Similarly, NUDCD1 knockdown overtly inhibited the invasion ability of the cells in comparison to the equivalent $\mathrm{Si}-\mathrm{NC}$ group (Figure 4E-4G).

\section{NUDCD1 modulates EMT in PC cells}

EMT is vital in the metastasis of cancer cells. The expression levels of $\mathrm{N}$-cadherin and vimentin were reduced whereas that of E-cadherin increased in NUDCD1 knockdown cell lines (Figure 5A-5H). Collectively, these results indicated that NUDCD1 knockdown may probably inhibit the metastasis of PC cells by modulating EMT.

\section{NUDCD1 silencing suppresses tumor growth in vivo}

We established a tumor xenograft model ro clarify more clearly the role of NUDCD1 in PC. The examination of subcutaneous tumorigenesis in nude mice revealed that tumor weight and volume were reduced remarkably following NUDCD1 knockdown (Figure 6A-6C). Western blot assay also revealed that NUDCD1 knockdown decreased the protein expression of vimentin and $\mathrm{N}$-cadherin and upregulated that of E-cadherin (Figure 6D-6G). These findings were consistent with those in vitro.

\section{DISCUSSION}

$\mathrm{PC}$ is one of the quickly growing malignant tumors $[12,13]$. The global occurrence rate of pancreatic cancer has increased rapidly in more than 10 years $[14,15]$. SLC16A is a common PC biomarker that is used in the classification of PC [16]. NUDCD1 plays a vital function in the pathogenesis of cancer $[17,18]$; however, the molecular mechanisms behind its functions in PC remain a mystery. NUDCD1 acts as a crucial component of apoptosis and immune responses, has been identified as an oncoprotein that is frequently activated or upregulated in various human cancers, and is known as an important kind of cancer antigen [18]. In the present study, NUDCD1 was upregulated in PC cell lines and tumor tissues, and its upregulation had a remarkable association with poor prognosis (Figure 1). Moreover, NUDCD1 silencing could inhibit the invasion, colony formation, migration, and proliferative abilities of PC cells (Figures 2, 4).

EMT is considered a major driver of metastasis in PDAC $[19,20]$. During invasion and metastasis, epithelial-derived cells lose the characteristics of epithelial cells, reduce intercellular adhesion, express markers of mesenchymal cells and enzymes that decompose extracellular matrix, which eventually leads to increased exercise and invasive abilities [21, 22]. In this study, we found that the downregulation of NUDCD1 could suppress the protein expression of vimentin and $\mathrm{N}$-cadherin and boost the expression levels of E-cadherin (Figure 5). These findings suggested that NUDCD1 could influence PC cell metastasis by regulating EMT. In addition, we conducted animal experiments to explore the role of NUDCD1 in tumorigenesis (Figure 6). As far as we know, the present study is the first to investigate the function of NUDCD1 in PC.

This study was limited because of the small sample size used to arrive at the conclusions. Further studies with larger sample sizes should be conducted to verify our findings.

In summary, the findings of this study showed that NUDCD1 silencing inhibits the invasion, colony formation, migration, and proliferation of PC cells and facilitates cell apoptosis through the EMT pathway. These data provide new insights into the identification of candidate molecular markers for the diagnosis and treatment of PC.

\section{Ethics statements}

The animal study was approved by the Institutional Animal Care and Use Committee of Wenzhou Medical University, China. The methods were performed according to the guidelines approved by the Institutional Review Board of Wenzhou Key Laboratory of Surgery, China.

\section{Availability of data and materials}

The additional data used to arrive at the conclusions can be obtained from the corresponding author upon reasonable request. 
A

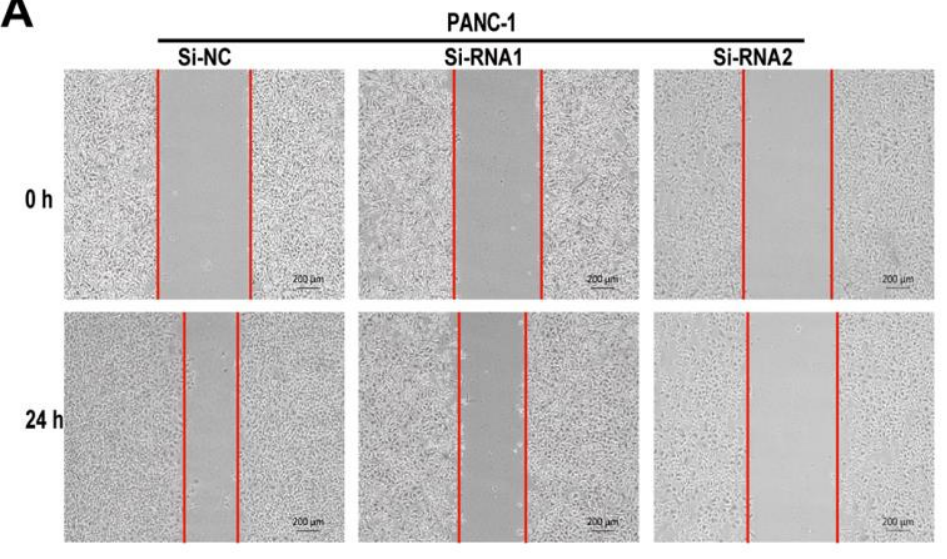

C

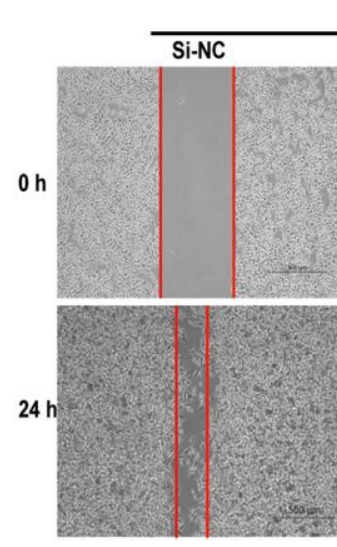

Patu8988
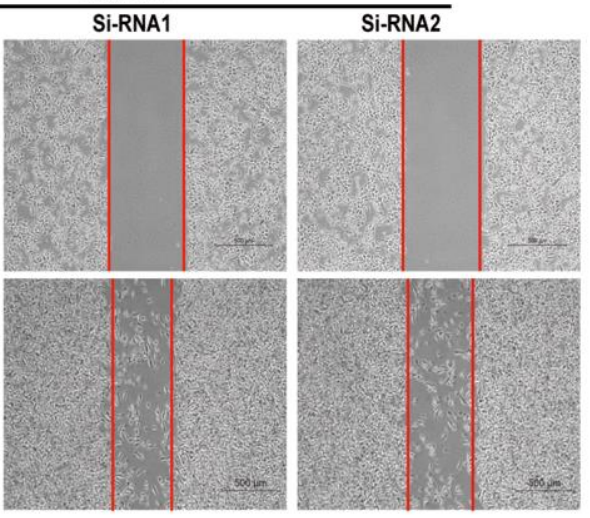

Si-RNA2
B

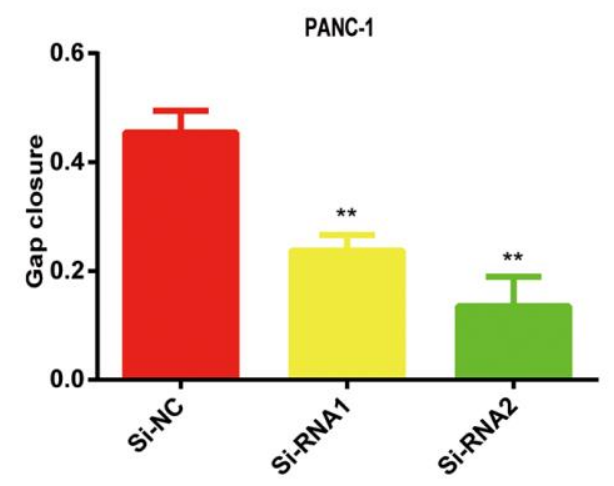

D

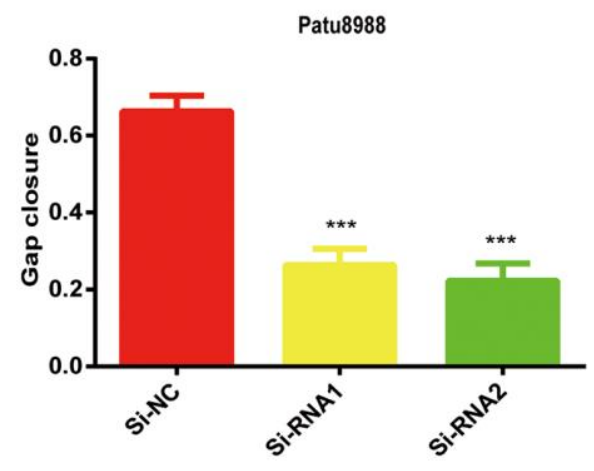

E

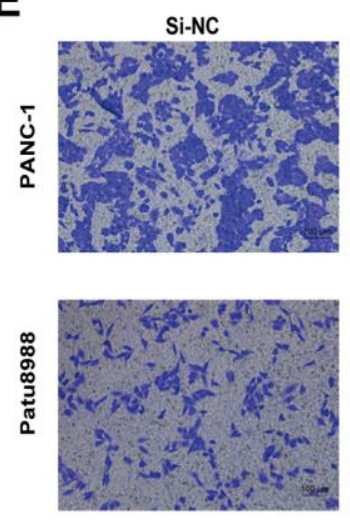

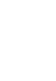


A

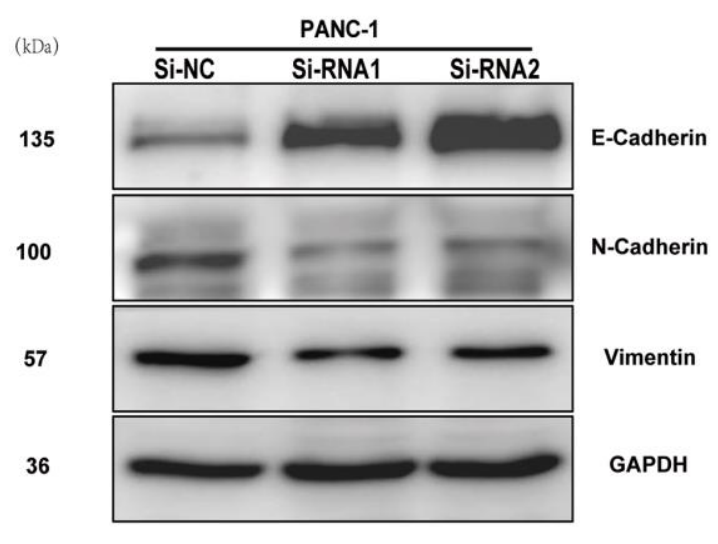

C

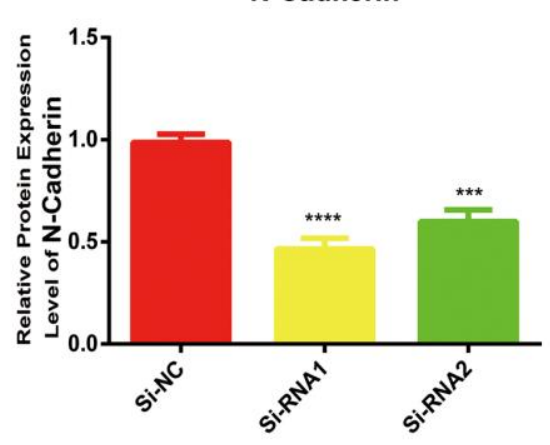

$\mathbf{E}$

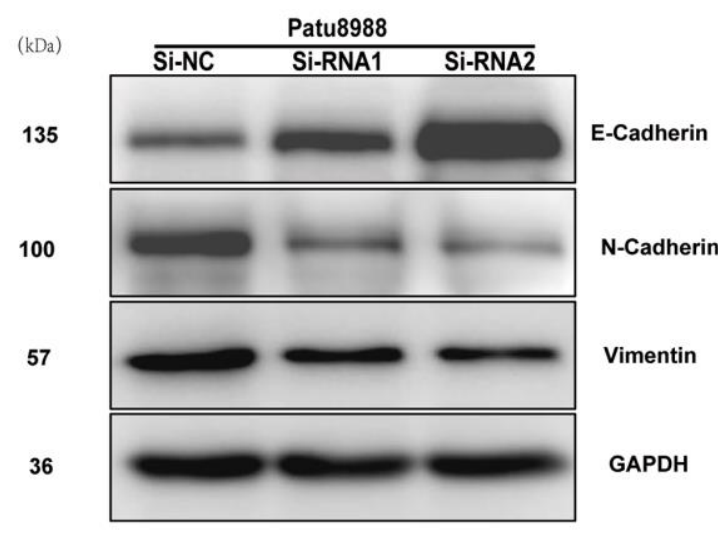

G

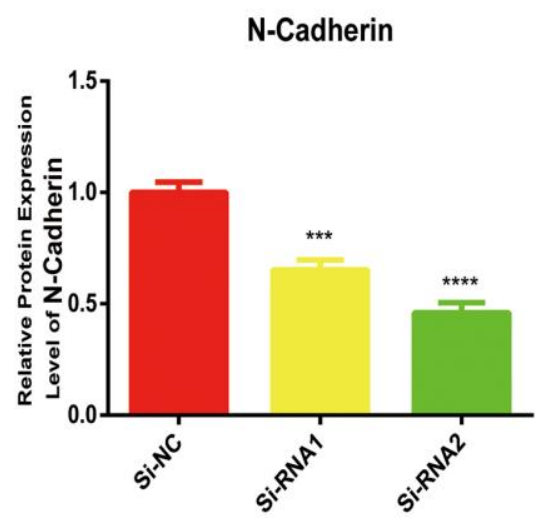

B

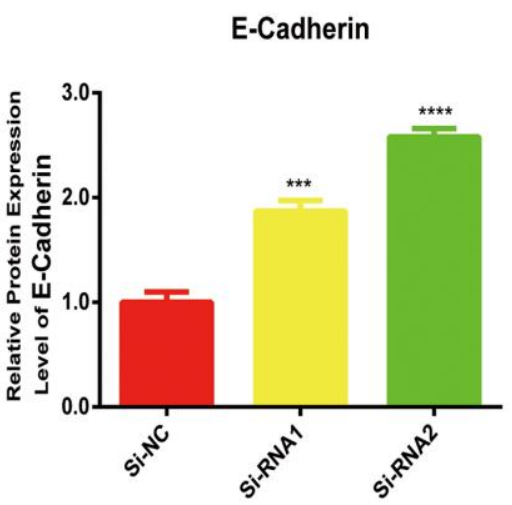

D

Vimentin

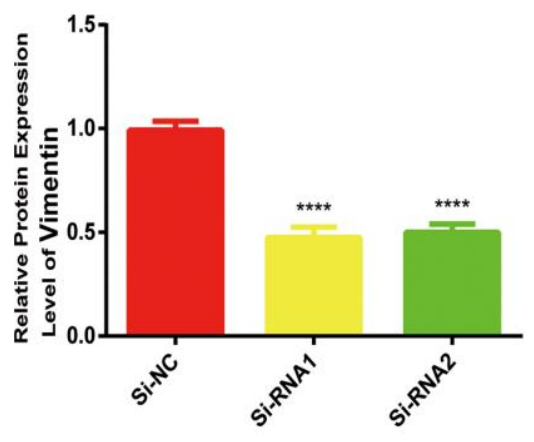

$\mathbf{F}$

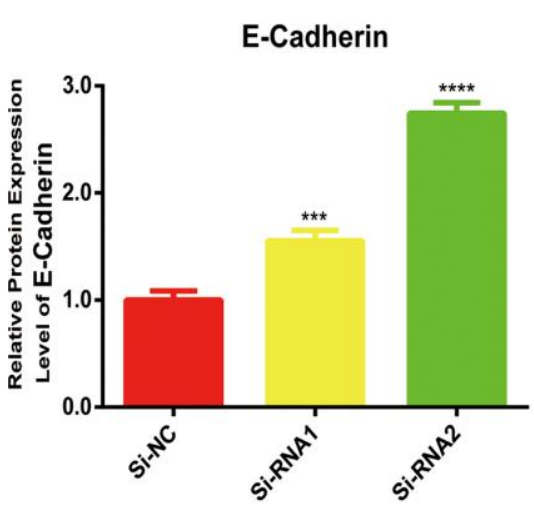

H

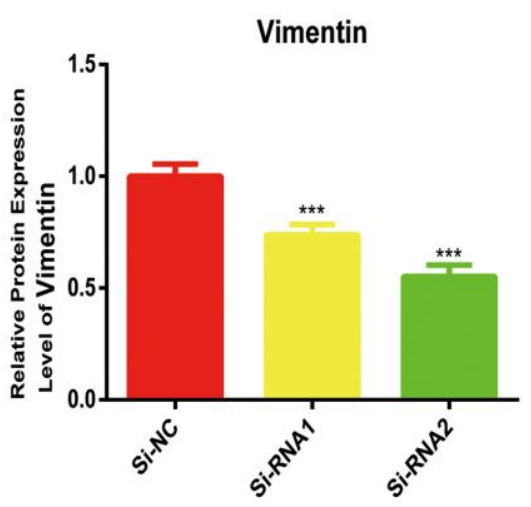

Figure 5. NUDCD1 knockdown inhibited the EMT process in PC cells. (A-D) NUDCD1 knockdown decreased the expression of Ncadherin and vimentin and upregulated the expression of E-cadherin in PANC-1 cells. (E-H) NUDCD1 knockdown decreased the expression of $\mathrm{N}$-cadherin and vimentin, and upregulated the expression of E-cadherin in Patu8988 cells. ${ }^{* * *} \mathrm{p}<0.001,{ }^{* * * *} \mathrm{p}<0.0001$. 
A

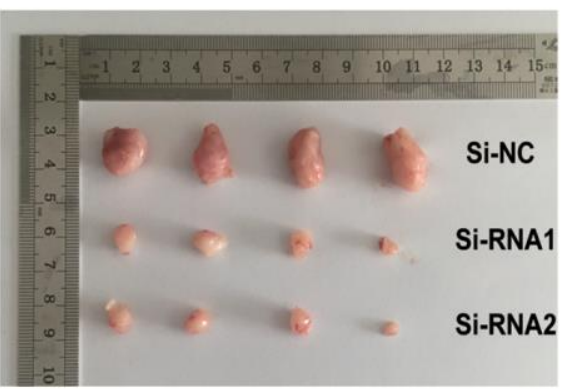

D

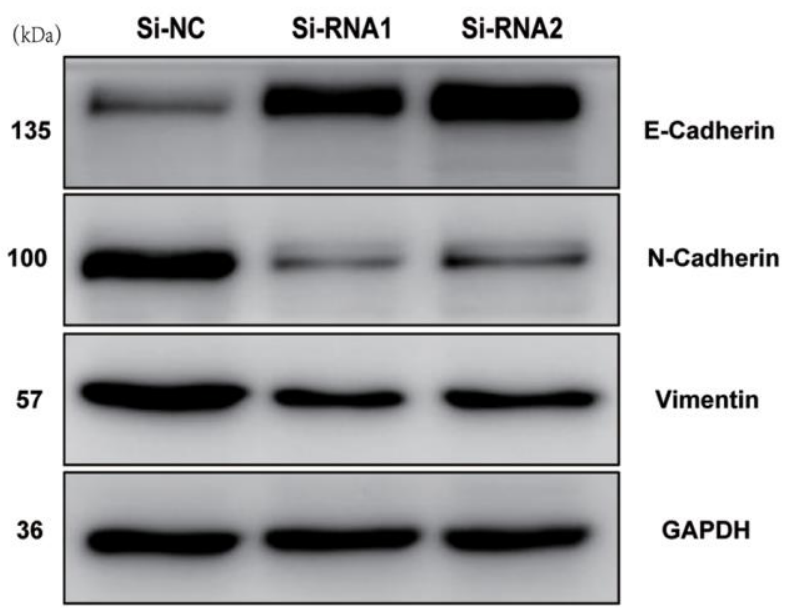

$\mathbf{F}$

N-Cadherin

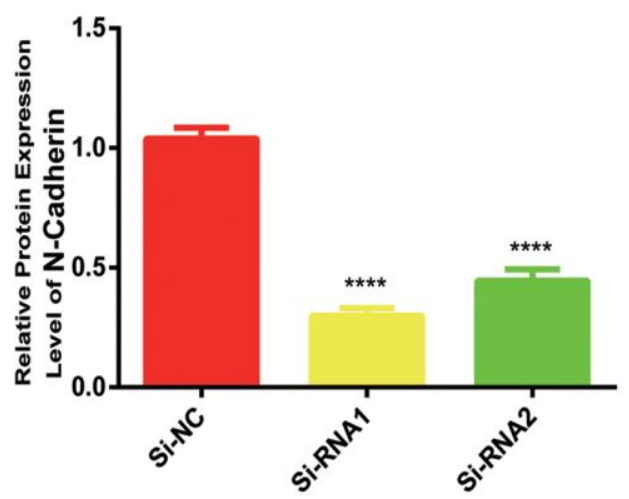

B
C
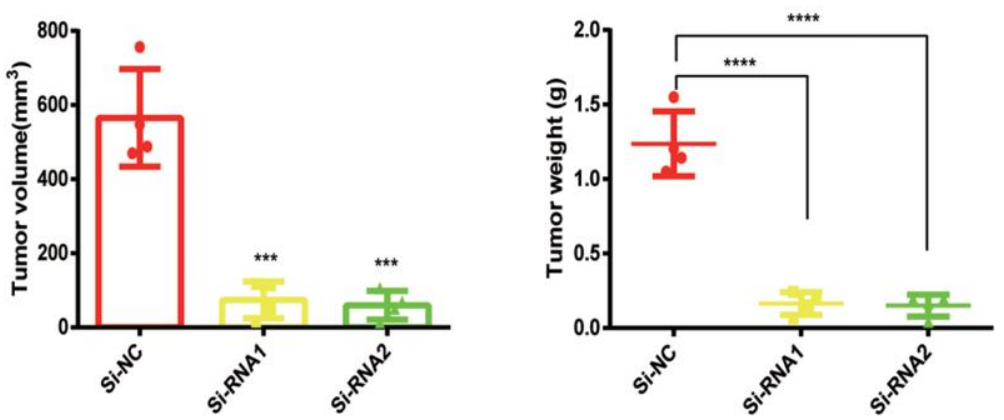

E

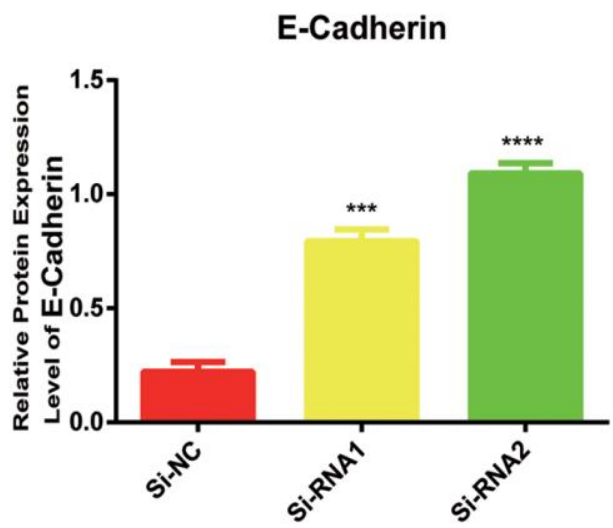

G

Vimentin

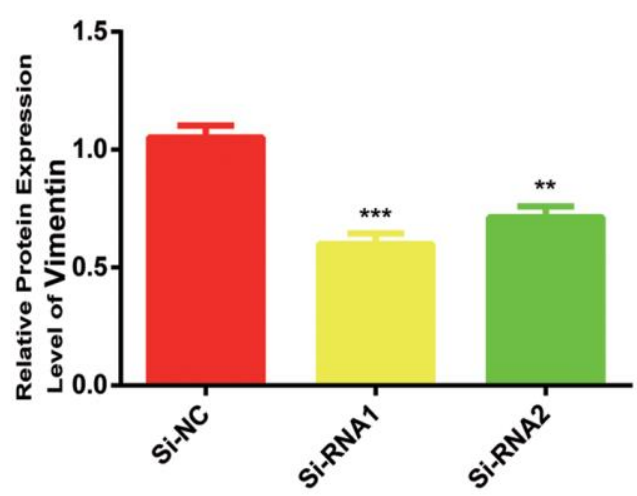

Figure 6. NUDCD1 knockdown inhibited the growth of PC in vivo. (A-C) NUDCD1 knockdown remarkably reduced tumor volume and weight. (D-G) NUDCD1 knockdown decreased the expression of $\mathrm{N}$-cadherin and vimentin and increased the expression of $\mathrm{E}$-cadherin in tumor tissue. ${ }^{* *} p<0.01,{ }^{* * *} p<0.001,{ }^{* * * *} p<0.0001$. 


\section{AUTHOR CONTRIBUTIONS}

H.Z. wrote the manuscript. C.S. conducted the main experiments. M.W. collected and analyzed the raw data. Y.G. and D.X. helped revise the article. H.J. and B.W. helped correct the article and supplementary experiments. Z.C. designed the whole work. All authors read and approved the final manuscript.

\section{CONFLICTS OF INTEREST}

The authors declare that they have no conflicts of interest.

\section{FUNDING}

This study was funded by Zhejiang Provincial Natural Science Foundation of China (No. LY14H140008) and Wenzhou Basic Research Project (No. Y2020993).

\section{REFERENCES}

1. Li Y, Chen Y, Li J, Zhang Z, Huang C, Lian G, Yang K, Chen S, Lin $Y$, Wang L, Huang $K$, Zeng L. Co-delivery of microRNA-21 antisense oligonucleotides and gemcitabine using nanomedicine for pancreatic cancer therapy. Cancer Sci. 2017; 108:1493-503.

https://doi.org/10.1111/cas.13267 PMID:28444967

2. Schober M, Jesenofsky R, Faissner R, Weidenauer $C$, Hagmann W, Michl P, Heuchel RL, Haas SL, Löhr JM. Desmoplasia and chemoresistance in pancreatic cancer. Cancers (Basel). 2014; 6:2137-54.

https://doi.org/10.3390/cancers6042137

PMID:25337831

3. Chen R, Lai LA, Sullivan $Y$, Wong $M$, Wang L, Riddell J, Jung L, Pillarisetty VG, Brentnall TA, Pan S. Disrupting glutamine metabolic pathways to sensitize gemcitabine-resistant pancreatic cancer. Sci Rep. 2017; 7:7950.

https://doi.org/10.1038/s41598-017-08436-6

PMID:28801576

4. Vila-Navarro E, Fernandez-Castañer E, Rovira-Rigau M, Raimondi G, Vila-Casadesus M, Lozano JJ, Soubeyran P, lovanna J, Castells A, Fillat C, Gironella M. MiR-93 is related to poor prognosis in pancreatic cancer and promotes tumor progression by targeting microtubule dynamics. Oncogenesis. 2020; 9:43.

https://doi.org/10.1038/s41389-020-0227-y PMID:32366853

5. Asselin-Mullen $P$, Chauvin A, Dubois ML, Drissi R, Lévesque $D$, Boisvert FM. Protein interaction network of alternatively spliced NudCD1 isoforms. Sci Rep. 2017; 7:12987. https://doi.org/10.1038/s41598-017-13441-w

PMID:29021621

6. He H, Dai J, Wang X, Qian X, Zhao J, Wang H, Xu D. NudCD1 affects renal cell carcinoma through regulating LIS1/Dynein signaling pathway. Am J Transl Res. 2018; 10:519-24.

PMID:29511446

7. Yan $Y$, Phan L, Yang $F$, Talpaz $M$, Yang $Y$, Xiong Z, Ng B, Timchenko NA, Wu CJ, Ritz J, Wang H, Yang XF. A novel mechanism of alternative promoter and splicing regulates the epitope generation of tumor antigen CML66-L. J Immunol. 2004; 172:651-60. https://doi.org/10.4049/jimmunol.172.1.651 PMID: 14688378

8. Yang XF, Wu CJ, McLaughlin S, Chillemi A, Wang KS, Canning C, Alyea EP, Kantoff P, Soiffer RJ, Dranoff G, Ritz J. CML66, a broadly immunogenic tumor antigen, elicits a humoral immune response associated with remission of chronic myelogenous leukemia. Proc Natl Acad Sci USA. 2001; 98:7492-97.

https://doi.org/10.1073/pnas.131590998

PMID:11416219

9. Song $F$, Chen $Q$, Rao W, Zhang R, Wang $Y, G e ~ H$, Wei Q. OVA66 promotes tumour angiogenesis and progression through enhancing autocrine VEGF-VEGFR2 signalling. EBioMedicine. 2019; 41:156-66.

https://doi.org/10.1016/j.ebiom.2019.02.051

PMID: $\underline{30833190}$

10. Sankowski R, Strohl JJ, Huerta TS, Nasiri E, Mazzarello AN, D’Abramo C, Cheng KF, Staszewski O, Prinz M, Huerta PT, Al-Abed Y. Endogenous retroviruses are associated with hippocampus-based memory impairment. Proc Natl Acad Sci USA. 2019; 116:25982-90.

https://doi.org/10.1073/pnas.1822164116

PMID:31792184

11. Kočevar N, Odreman F, Vindigni A, Grazio SF, Komel R. Proteomic analysis of gastric cancer and immunoblot validation of potential biomarkers. World J Gastroenterol. 2012; 18:1216-28.

https://doi.org/10.3748/wjg.v18.i11.1216

PMID:22468085

12. Huang $Q$, Zhang J, Peng J, Zhang $Y$, Wang $L, W u J$, Ye $L$, Fang $C$. Effect of baicalin on proliferation and apoptosis in pancreatic cancer cells. Am J Transl Res. 2019; 11:5645-54.

PMID:31632536

13. Shen T, Zhou L, Shen H, Shi C, Jia S, Ding GP, Cao L. Prognostic value of programmed cell death protein 1 expression on CD8+ $T$ lymphocytes in pancreatic cancer. Sci Rep. 2017; 7:7848. 
https://doi.org/10.1038/s41598-017-08479-9

PMID:28798308

14. Gordon-Dseagu VL, Devesa SS, Goggins M, Stolzenberg-Solomon R. Pancreatic cancer incidence trends: evidence from the Surveillance, Epidemiology and End Results (SEER) population-based data. Int J Epidemiol. 2018; 47:427-39. https://doi.org/10.1093/ije/dyx232 PMID:29149259

15. Kunk PR, Bauer TW, Slingluff $\mathrm{CL}$, Rahma OE. From bench to bedside a comprehensive review of pancreatic cancer immunotherapy. J Immunother Cancer. 2016; 4:14.

https://doi.org/10.1186/s40425-016-0119-z

PMID:26981244

16. Yu S, Wu Y, Li C, Qu Z, Lou G, Guo X, Ji J, Li N, Guo M, Zhang $M$, Lei $L$, Tai $S$. Comprehensive analysis of the SLC16A gene family in pancreatic cancer via integrated bioinformatics. Sci Rep. 2020; 10:7315. https://doi.org/10.1038/s41598-020-64356-y PMID:32355273

17. Wang RJ, Wang N, Cui G, Chen Y, Zhong H, Tang J. The impact of NudCD1 on renal carcinoma cell proliferation, migration, and invasion. Eur Rev Med Pharmacol Sci. 2018; 22:671-77.

https://doi.org/10.26355/eurrev 201802 14292 PMID:29461594

18. Han B, Zhang YY, Xu K, Bai Y, Wan LH, Miao SK, Zhang KX, Zhang HW, Liu Y, Zhou LM. NUDCD1 promotes metastasis through inducing EMT and inhibiting apoptosis in colorectal cancer. Am J Cancer Res. 2018; 8:810-23.

PMID:29888104

19. Title AC, Hong SJ, Pires ND, Hasenöhrl L, Godbersen S, Stokar-Regenscheit N, Bartel DP, Stoffel M. Genetic dissection of the miR-200-Zeb1 axis reveals its importance in tumor differentiation and invasion. Nat Commun. 2018; 9:4671. https://doi.org/10.1038/s41467-018-07130-z PMID: $\underline{30405106}$

20. Akimoto N, Nakamura K, Hijioka H, Kume K, Matsumura $Y$, Sugiura T. Transfection of T-Box Transcription Factor BRACHYURY and SOX2 Synergistically Promote SelfRenewal and Invasive Phenotype in Oral Cancer Cells. Int J Mol Sci. 2018; 19:3620.

https://doi.org/10.3390/ijms19113620

PMID:30453543

21. Cascione M, De Matteis V, Toma CC, Leporatti S. Morphomechanical Alterations Induced by Transforming Growth Factor- $\beta 1$ in Epithelial Breast Cancer Cells. Cancers (Basel). 2018; 10:234. https://doi.org/10.3390/cancers10070234 PMID:30012949

22. Shibata S, Hayashi R, Okubo T, Kudo Y, Baba K, Honma $Y$, Nishida K. The secretome of adipose-derived mesenchymal stem cells attenuates epithelialmesenchymal transition in human corneal epithelium. Regen Ther. 2019; 11:114-22.

https://doi.org/10.1016/i.reth.2019.06.005

PMID:31312693 